\title{
Primeras aproximaciones al registro zooarqueológico de Pajchela Núcleo, Puna de Jujuy: un acercamiento a las prácticas de manejo y consumo de animales en un contexto agro-pastoril
}

Jesica Carreras"
Recibido:

19 de febrero de 2019

Aceptado:

10 de septiembre de 2019

\section{Resumen}

Este artículo tiene como objetivo presentar los resultados preliminares del análisis del conjunto zooarqueológico proveniente del recinto 1 del sitio Pajchela Núcleo, ubicado en la cuenca superior del río Grande de San Juan en la Puna jujeña, Argentina. Propongo las primeras interpretaciones acerca de las prácticas domésticas relacionadas con la preparación de alimentos, focalizadas en el manejo y el consumo de animales entre los siglos XIV y XV, durante el periodo de Desarrollos Regionales Tardío en el sitio. La muestra está compuesta por un total de 1.590 especímenes óseos, de los que pudieron ser identificados un total de 662 (42\%), mientras que los 928 fragmentos restantes permanecen como no identificados (58\%). El conjunto general presenta una baja diversidad taxonómica. El taxón más abundante es Artiodactyla, seguido por Camelidae. Aunque se encuentra emplazado en un contexto agrícola, el recinto presenta una lógica de manejo de los rebaños y las prácticas vinculadas con la obtención y consumo de animales que responde a una estrategia pastoril, en la que la producción secundaria, posiblemente de fibra y la capacidad de transporte, jugaron roles importantes. Considero, entonces, que la formación del registro arqueofaunístico responde a una lógica propia de un sistema agro-pastoril complejo.

First approaches to the zooarchaeological record of Pajchela Núcleo, Puna of Jujuy: An approach to animal management and consumption practices in an agro-pastoral context

\footnotetext{
Abstract

In this article we present the preliminary results of the zooarchaeological analysis of the assemblage recovered in Pajchela Núcleo, an archaeological site located in the upper basin of the río Grande de San Juan, in the Puna of Jujuy, Argentina. I suggest interpretations about the domestic practices related to food preparation, focusing on the management and consumption of animals between the fourteenth and fifteenth

\section{Palabras clave}

Agro-pastoralismo Camélidos Cusi Cusi Periodo de Desarrollos Regionales Zooarqueología
Keywords

Agro-pastoralism Camelids Cusi Cusi

Regional Developments period Zooarchaeology 
centuries, during the Late Regional Developments Period. The sample is composed of 1,590 bone specimens, 662 (42\%) of them could be identified, while the remaining 928 fragments were quantified as unidentified (58\%). The assemblage presents a low taxonomic diversity. The most abundant taxon was Artiodactyla, followed by Camelidae. Although located in an agricultural context, the enclosure presents a structure consistent with herd management, and practices related to the obtention and consumption of animals, which respond to a pastoral strategy in which secondary production, possibly of fiber, and transport capacity, plays an important role. Therefore, I believe that the formation of the archaeological record responds to the logic of a complex agro-pastoral system.

\section{Introducción}

La forma en la que preparamos los alimentos, tanto hoy como en el pasado, es un fenómeno complejo que está determinado por diversos factores. Tradicionalmente, la arqueología de la alimentación ha encarado la problemática utilizando conceptos que remiten a la subsistencia, el análisis de rendimiento calórico, los modelos de productividad, la dieta, la nutrición y la accesibilidad e intercambio de recursos. Sin embargo, los humanos en tanto somos seres sociales, no solamente comemos alimentos y nutrientes sino fundamentalmente comida y el evento alimentario está siempre situado tanto en una sociedad determinada como en un espacio y un tiempo culturalmente específico. Siguiendo lo planteado por Pazzarelli (2008), propongo un acercamiento a una "arqueología de la comida", teniendo en cuenta que la alimentación es un hecho complejo, multidimensional, que entrelaza sustancias y personas, pero también historias y lugares, tiempos y maneras de hacer las cosas. La alimentación, entonces, se entiende como un macroproceso que incluye tanto el consumo de alimentos como las prácticas relativas a su preparación y cocción (Ávido, 2012; De Feo, Fernández y Raviña, 2007; Goody, 1995; Samuel, 1996) y que tienen como escenario la vivienda. Así entendidas, las prácticas alimentarias integran no solo qué se comió sino también cómo se comió (Marschoff, 2007).

Ahora bien, esta investigación supone un primer acercamiento a una parte del fenómeno multidimensional de la alimentación a partir del análisis del conjunto zooarqueológico del recinto 1 del sitio Pajchela Núcleo. Se proponen interpretaciones preliminares acerca de las prácticas domésticas vinculadas a la preparación de alimentos, enfatizando cómo se manejaban y se consumían los animales entre los siglos XIV y XV durante el periodo de Desarrollos Regionales Tardío en este sitio. Además, se exploran aspectos relacionados con la formación del conjunto y su relación con actividades pastoriles. Al mismo tiempo y con el fin de interpretar las primeras tendencias obtenidas, se contrasta la evidencia etnográfica de prácticas de manejo y consumo de recursos faunísticos disponible para pastores actuales. Finalmente, se comparan las tres ocupaciones identificadas para el recinto, investigando aquellos cambios y continuidades que se reflejan en la muestra zooarqueológica para interpretar la manera en que se manejaban los animales.

Se considera que, aunque el recinto se encuentra emplazado en un contexto agrícola, el manejo de los rebaños y las prácticas vinculadas con la obtención y consumo de animales responden a una estrategia pastoril en la que la producción de otros productos además de la carne tuvo un rol importante. De esta manera, la formación del registro zooarqueológico respondería a una lógica propia de un sistema agro-pastoril en la que ambas actividades económicas se combinan. Se propone que al igual que en las viviendas pastoriles actuales, los camélidos fueron sacrificados, procesados y principalmente 
consumidos y descartados en el espacio doméstico (Tomka, 1994; Yacobaccio, 1994; Yacobaccio, Madero y Malmierca, 1998).

\section{Características generales del área de estudio}

La zona de estudio se localiza en los departamentos de Rinconada y Santa Catalina de la provincia de Jujuy, Argentina (Figura 1). Esta área se encuentra situada dentro de la Puna de Jujuy, entendida como la porción nororiental de la provincia que abarca las cuencas de drenaje atlántico del río Grande de San Juan, Yavi y Sansana y las cuencas endorreicas de Pozuelos y Miraflores-Guayatayoc (Albeck y Ruiz, 2003). Este trabajo se centra en la cuenca superior del río Grande de San Juan, área que limita con la Cordillera de Lípez al Oeste, el Volcán Granada hacia el Sur, la divisoria de aguas emplazada en las alturas mayores a $4.000 \mathrm{~m}$ s.n.m. hacia el Este y, finalmente, el angosto del Río San Juan hacia el Norte, localizado a $2 \mathrm{~km}$ de la localidad de San Juan de Oro. Esta zona corresponde a las nacientes del río y se caracteriza por la presencia de quebradas profundas con orientación oeste-este que bajan de la Cordillera de Lípez y desembocan en la quebrada principal del río Grande de San Juan. El poblado actual de mayor tamaño es Cusi Cusi. El clima del área presenta las características generales del ambiente de Puna seca, un clima frío y seco con una alta evapotranspiración, estacionalidad bien marcada y gran amplitud térmica diaria (Saravia, 1960). En la Puna seca las precipitaciones son escasas, oscilando entre oscilando entre 200 y $400 \mathrm{~mm}$ anuales (Buitrago, 1999). Este ambiente se caracteriza por presentar un paisaje desértico $\mathrm{y}$ fragmentado, con una escasa cobertura vegetal dominada por especies arbustivas.

La vegetación corresponde a la provincia fitogeográfica puneña (Cabrera, 1976) con predominio de pajonales de altura. Se caracteriza principalmente por la presencia de arbustos leñosos bajos como la tola (Lephidophyllum quadrangulare), cactus aislados (Opuntia floccosa) y en los sectores más secos la yareta (Azorella compacta). También hay cardones (Echinopsis atacamensis) y paja ichu (Festuca ortophylla). Con respecto a la fauna, se encuentra una especie de camélido doméstico, la llama (Lama glama), y dos especies de camélidos silvestres, el guanaco andino (Lama guanicoe cacsilensis) y la vicuña (Vicugna vicugna), a los que se suma la taruca o venado andino (Hypocamelus antisensis). Hay también varias especies de roedores como la vizcacha (Lagidium viscacia), la chinchilla (Chinchilla laniger) y varios otros pequeños. Completan la fauna varias aves, el puma (Felis concolor) y dos felinos menores: el gato andino (Oreailurus jacobita) y el gato del pajonal (Oncifelis colocolo).

Es importante resaltar que las investigaciones arqueológicas en la región no fueron sistemáticas y tuvieron un desarrollo bastante reciente en comparación con otras zonas cercanas, aunque existen algunos trabajos previos (De Feo, Fernández y Raviña, 2001, 2007; Nielsen, Ávalos, Ávila, Guagliardo y López, 2008). Desde el año 2010, el equipo del Dr. José María Vaquer se encuentra realizando estudios en la cuenca superior del río Grande de San Juan, específicamente en Cusi Cusi (departamento de Rinconada, Jujuy). Aquí, se realizaron trabajos de prospección, registro y excavación de sitios arqueológicos a partir de lo que se han registrado más de 100 sitios arqueológicos (Pey, 2015; Vaquer, Gerola, Carboni, y Bonelli, 2014; Vaquer, Zuccarelli, Pey y Cámera, 2014). Pajchela Núcleo y Casas Quemadas son los únicos sectores donde se han relevado recintos habitacionales. Teniendo en cuenta la información disponible, Casas Quemadas y Pajchela Núcleo forman parte de un sistema agrícola y serían el producto de la imposición de una lógica agrícola sobre un trasfondo de pastores (Pey, 2015).

\section{El sitio}

Pajchela Núcleo (desde ahora PJN) es un sitio compuesto por pocos recintos habitacionales (no más de cinco) y estructuras agrícolas y de manejo del agua, 


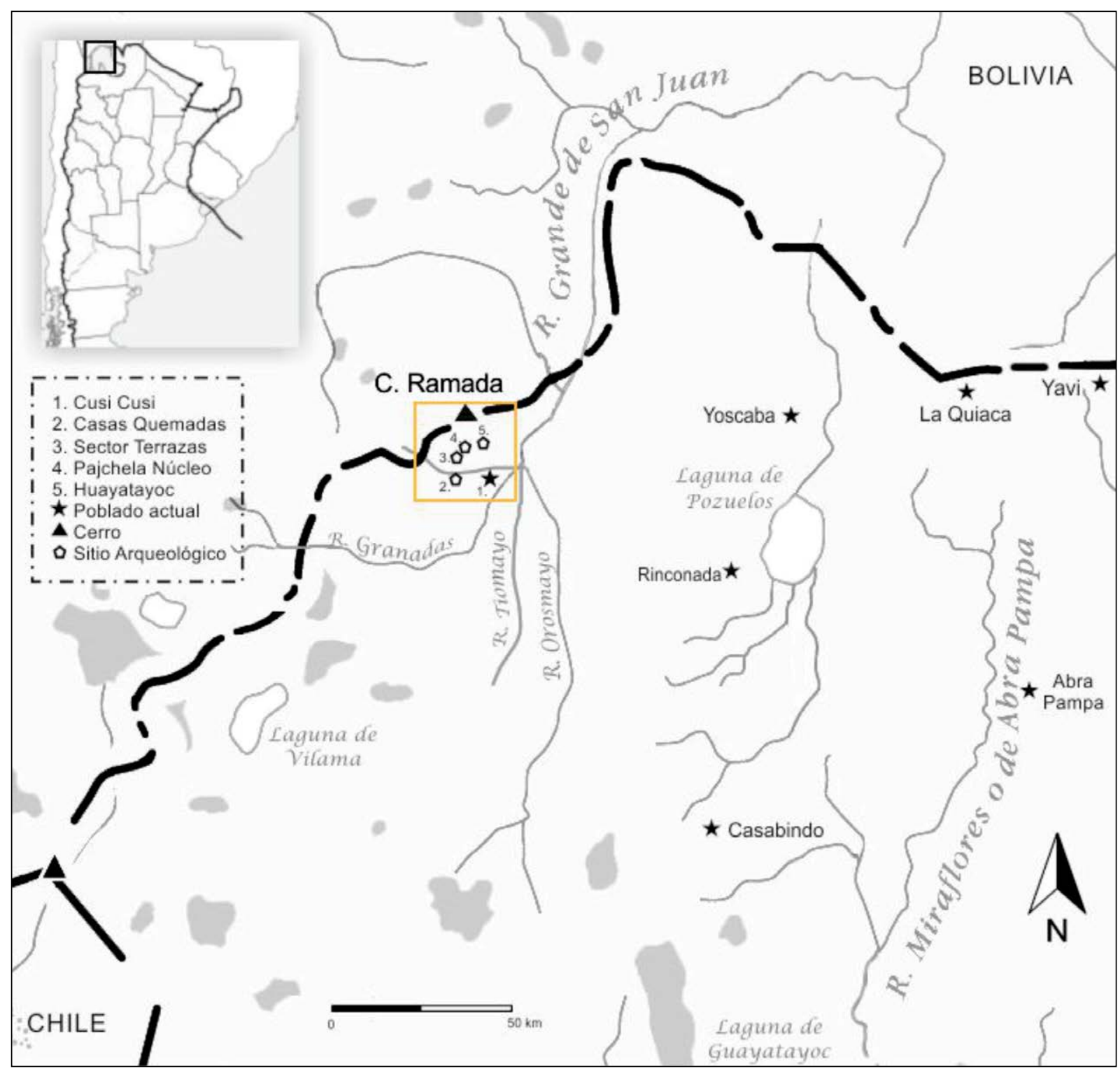

Figura 1. Localización de la zona de estudio.

conformando un sistema productivo complejo (Figura 2). El patrón de asentamiento se completa con un montículo plataforma artificial construido en tres niveles en la terraza del río sobre un afloramiento rocoso. Esta estructura se encuentra conformada por 15 cámaras de piedra en distintos estados de conservación.

El Recinto 1 de este sitio posee una planta rectangular y mide 3,64 m por 3,30 m. Los muros presentan un buen estado de conservación, con excepción del muro noreste que se encuentra derrumbado y solamente se conservaron los cimientos. Los muros restantes presentan hilada simple, con aparejo rústico y trabajo natural. La cuadrícula 1 , única analizada hasta el momento, se localizó en la mitad noroeste del recinto y mide 1,80 m por 2,42 m (Figura 3). La excavación se realizó por niveles naturales, identificando una secuencia estratigráfica compuesta por 12 unidades de proveniencia (UP).

A partir de las investigaciones realizadas en el sitio, se ha interpretado la existencia de dos ocupaciones sobre la base de la secuencia estratigráfica y las relaciones establecidas con 


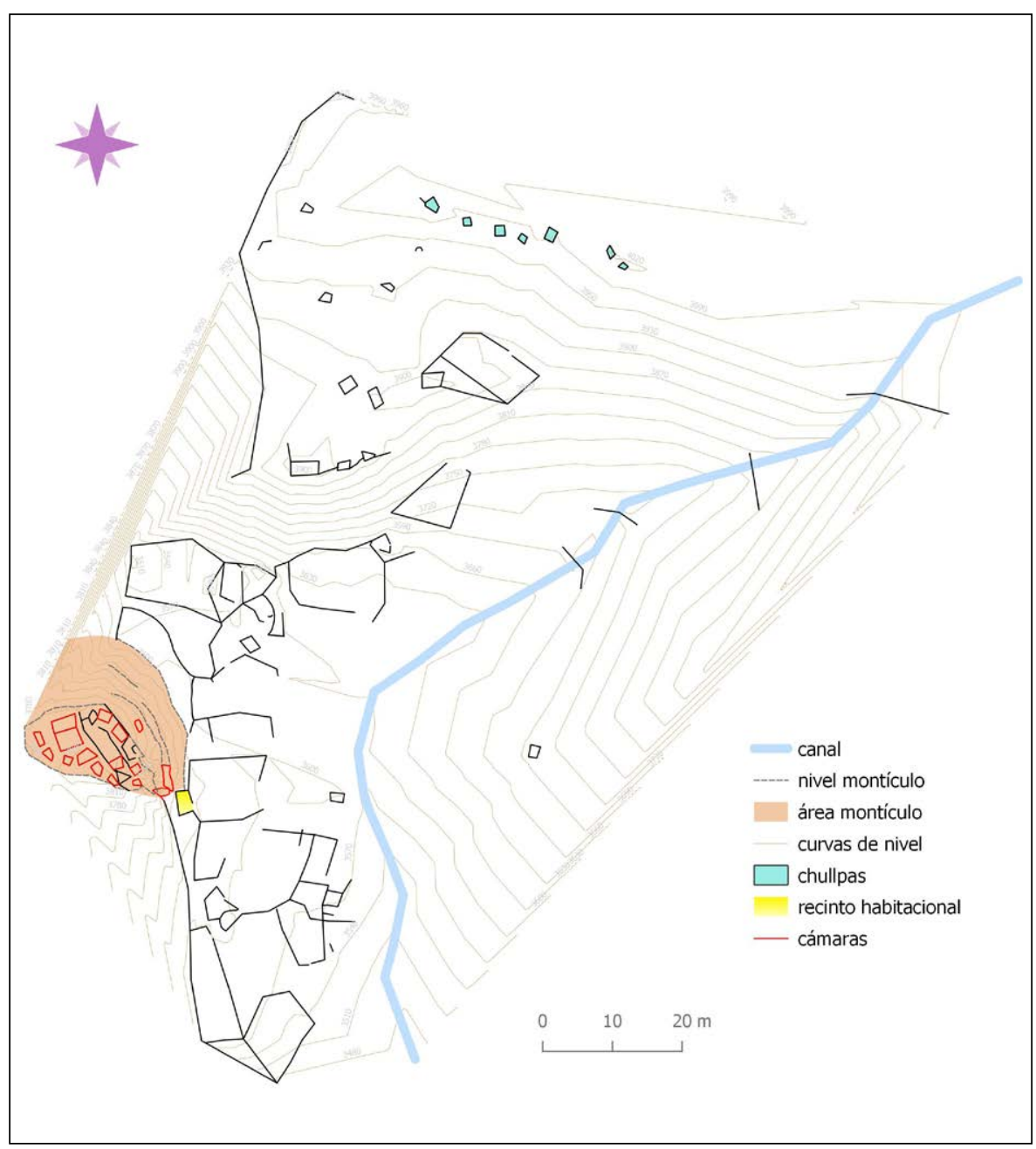

Figura 2. Plano de Pajchela Núcleo.

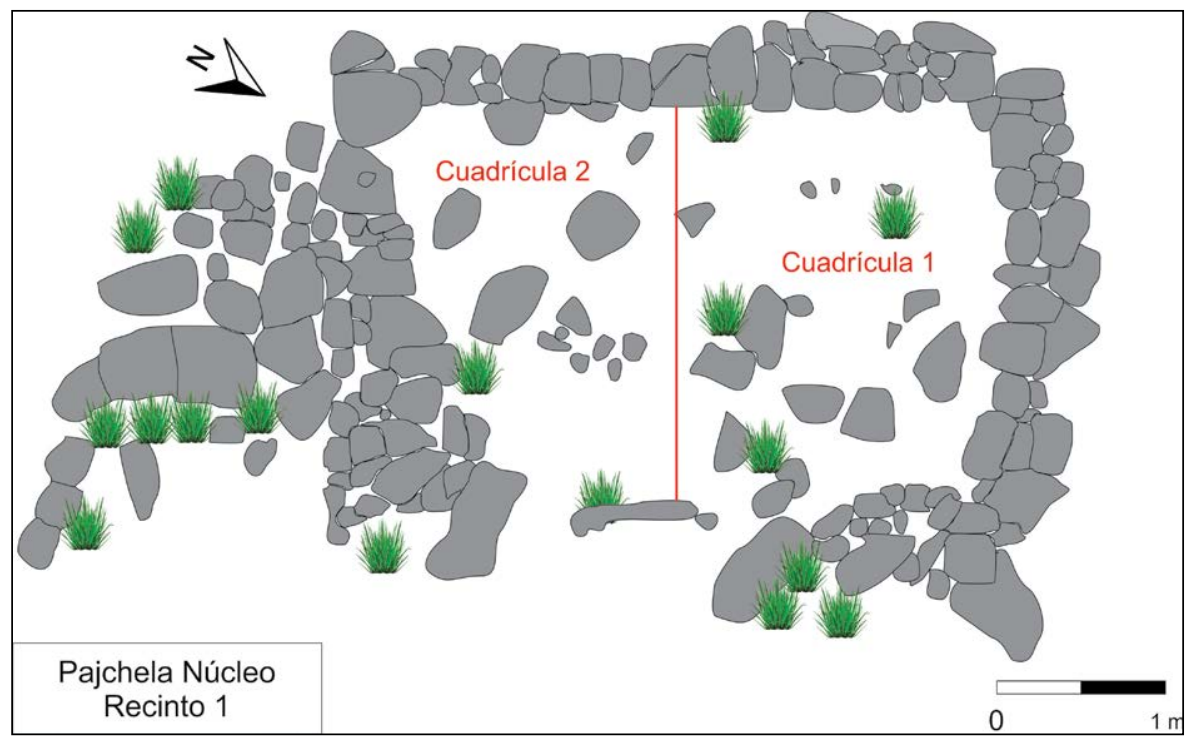

Figura 3. Planta del recinto 1 de PJN, mostrando las cuadrículas excavadas. 
los materiales hallados. Se ha establecido una ocupación inicial del recinto que abarca las UP 5, 10, 13, 11 y 14 . Las UP 11 y 14 corresponden a eventos de combustión. La primera se trata de un fogón en cubeta, localizado en la esquina noreste de la cuadrícula excavada. Mientras que la UP 14 es un lente de ceniza que se encuentra separado de la UP 11 por una roca y resulta imposible determinar si se trata del mismo o de diferentes eventos. Ambos fogones fueron datados. Los fechados obtenidos permiten ubicar la primera ocupación entre los siglos XIV y XV en pleno periodo de Desarrollos Regionales Tardío (Figura 4): la UP 11 otorgó un fechado de $600 \pm 40$ años AP [cal A.D. 1.389: cal A.D. 1422] (LP-3544, carbón vegetal, $\delta^{13} \mathrm{C}$ (estimado): $-24 \pm 2 \%$ ); mientras que la UP 14, $510 \pm 40$ años AP [cal A.D. 1.420: cal A.D. 1.452] (LP-3551, carbón vegetal, $\delta^{13} \mathrm{C}$ (estimado): $-24 \pm 2 \%$ ).

Si bien los resultados presentados en este trabajo son preliminares debido a que aún queda la mitad del recinto por analizar, ellos constituyen un acercamiento a las prácticas de los habitantes de PJN relacionadas con el consumo de animales y muestran una serie de tendencias a profundizar. La muestra, si bien sesgada como ya se ha indicado, puede ser considerada representativa de la forma de habitar este espacio, ya que se trata de un sitio en el que los recintos habitacionales no son abundantes. Sin embargo, futuras excavaciones permitirán ampliar la muestra. Sobre la base de la muestra zooarqueológica, se definieron variables para los dos momentos de ocupación identificados, relativas a la preservación posdepositacional, la identificación taxonómica, la frecuencia de unidades anatómicas, las modificaciones óseas y las edades de muerte, definiendo estas variables para los dos momentos de ocupación descritos. Así se buscan identificar las prácticas humanas que intervinieron en la formación del registro zooarqueológico del recinto 1.

\section{Materiales y métodos}

\section{Óseo}

Como se mencionara, entonces, la muestra analizada proviene de la cuadrícula 1 del recinto 1 de PJN. Está compuesta por un total de 1.590 especímenes óseos. Con el fin de realizar las primeras comparaciones e identificar cambios y continuidades en el uso de los animales en el recinto, se identificaron tres conjuntos arqueofaunísticos:

» Conjunto 1 (C1) (abarca las UP 1 y 2): se trata del conjunto hallado en superficie, por encima del derrumbe de los muros del recinto. Está conformado por 39 especímenes óseos.

» Conjunto 2 (C2) (abarca las UP 3, 4 y 16): constituye la ocupación final del recinto y se encuentra por debajo del derrumbe de los muros. No es posible precisar la temporalidad de este conjunto. Hasta el momento, no se han encontrado fogones asociados. Sin embargo, en base al análisis del material de estas UP, se ha podido identificar que se trata de una ocupación distinta de la fundacional. El conjunto arqueofaunístico está constituido por 262 especímenes óseos.

» Conjunto 3 (C3) (abarca las UP 5, 13, 10, 11, 12 y 14): se trata del conjunto correspondiente a la ocupación inicial del recinto. Está asociado a dos fogones, con fechados que sitúan la ocupación entre 1320 y 1450 A.D. El conjunto arqueofaunístico está compuesto por 1.289 especímenes óseos.

La selección de variables tenidas en cuenta para el presente trabajo, se tomaron en base a aquellas seleccionadas por Miyano (2018) para la identificación de cambios y continuidades en las estrategias vinculadas a la obtención, manejo, aprovechamiento y consumo de animales en los conjuntos arqueofaunísticos provenientes de Palo Blanco 


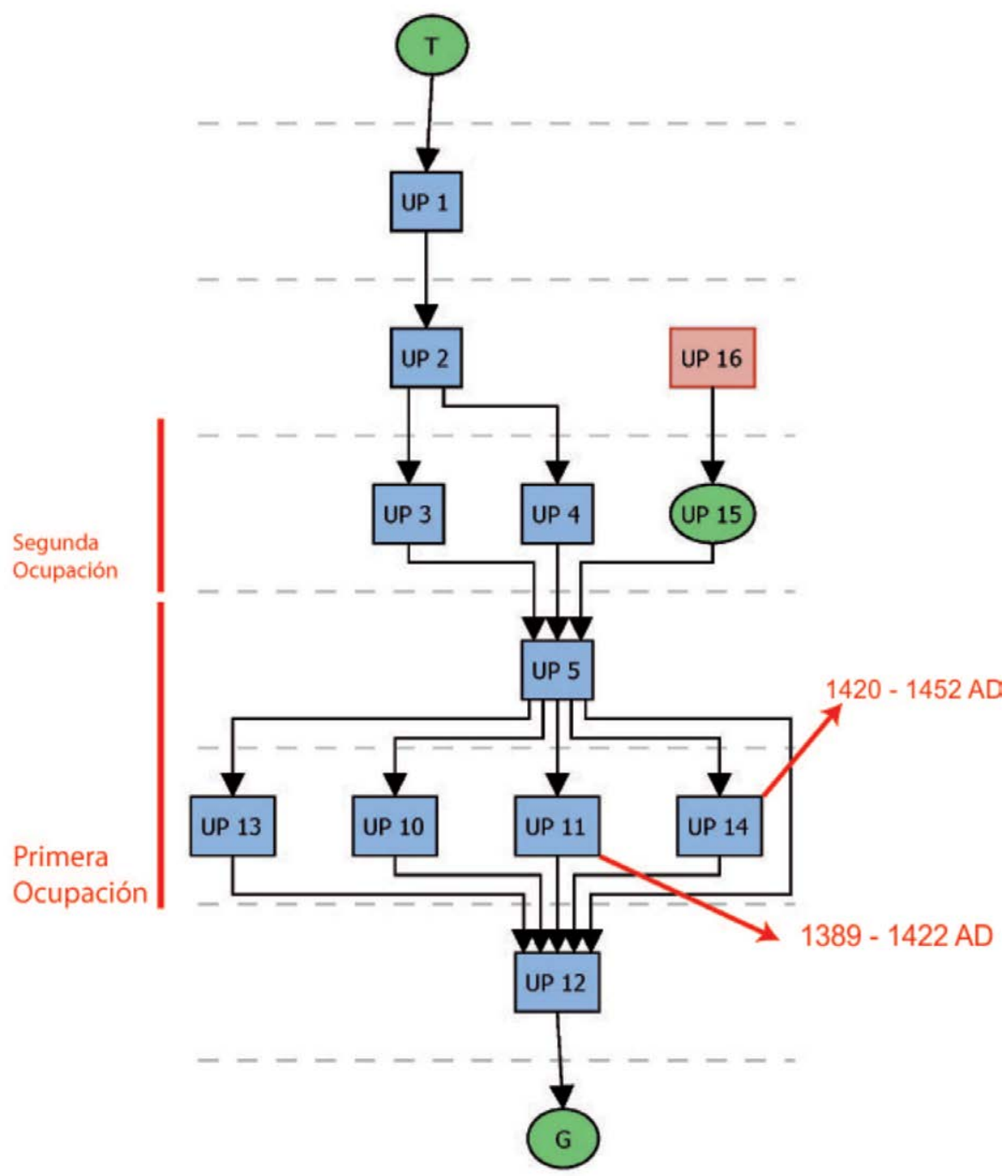

Figura 4. Matriz estratigráfica de la cuadrícula 1 mostrando las ocupaciones y los fechados.

(valle de Fiambalá, Catamarca). En los tres conjuntos, entonces, se tuvieron en cuenta las mismas variables. En primer lugar, se calculó el Número Total de Especímenes óseos (NSP = NISP + NID), los No Identificados (NID) y el Número de Especímenes Óseos Identificados (NISP). Luego, se registraron aquellas modificaciones observadas en el conjunto. Por un lado, se analizaron las marcas de origen antrópico relacionadas al procesamiento de los animales (Lyman, 1994; Mengoni Goñalons, 1999), así como también aquellas marcas que sugieren la formatización de restos óseos (Izeta, 2007; Izeta, Cattáneo, Scattolin y Cortés, 2013). En relación con el grado de integridad de la muestra, se consideraron los estadios de meteorización del NISP de acuerdo con Behrensmeyer (1978) y la presencia de marcas naturales producidas por carnívoros, roedores y raíces (Mengoni Goñalons, 1999). También se registró el color de los especímenes óseos del Número Total de Especímenes óseos (NSP = NISP + NID) para dar cuenta de acción térmica, siguiendo cuatro estadios: sin alteración térmica, quemado, carbonizado y calcinado (Mengoni Goñalons, 1999).

A nivel taxonómico se procedió a la identificación anatómica y taxonómica utilizando muestras comparativas del IDA (Instituto de Arqueología, Facultad de Filosofía y 
Letras, Universidad de Buenos Aires). Aquellos especímenes identificados se asignaron a diferentes categorías taxonómicas en base a la presencia de zonas diagnósticas (Mengoni Goñalons, 1999). Estos especímenes identificados constituyen el Número de Especímenes Identificados por Taxón (NISP) (Lyman, 1994). Los restos óseos que debido a su fragmentación no pudieron ser identificados, fueron contabilizados como no identificados (NID) (Mengoni Goñalons, 1999).

Para diferenciar camélidos silvestres de domesticados existen diversos métodos que pueden utilizarse. En este caso se han utilizado la medición osteométrica de falanges proximales ya que no se contaba con metapodios enteros ni con mandíbulas con dientes para complementar el análisis. Para la medición de las falanges, las medidas de referencia corresponden a un guanaco de las Cumbres Calchaquíes, Salta; una vicuña de Abra Pampa, Jujuy; vicuña de Abra Pampa, Jujuy y llama de Rinconada, Jujuy (Mercolli, comunicación personal, 2018). Para ser sometidos a osteometría los huesos deben presentar un buen estado de conservación, no estar termoalterados y mostrar madurez osteológica (estar fusionados) (Elkin, 1995; von den Driesch, 1976).

Para el subconjunto Camelidae se analizaron diversas variables: abundancia de partes esqueletarias, evaluando los aportes cárnicos y de médula de cada uno, y construcción de perfiles etarios. En relación con la construcción de perfiles etarios, se tuvo en cuenta la fusión ósea, ya que no se dispone de mandíbulas ni de maxilares en buen estado de conservación. Los estados de fusión se dividieron en tres segmentos: temprana, intermedia y tardía. Los huesos del esqueleto que se tomaron en consideración fueron la escápula (coracoides), epífisis proximales de las primeras falanges, sectores distales y proximales de huesos largos en general y pelvis (acetábulo). Así, la construcción de los perfiles etarios se realizó a partir de las frecuencias de NISP (F o NF) para cada una de las tres etapas mencionadas anteriormente.

Entonces, en relación con la abundancia anatómica de Camelidae se utilizaron diversas variables. Por un lado, se calculó el Número Mínimo de Elementos (MNE) combinando el método de zonas diagnósticas (Mengoni Goñalons, 1999) y la suma de fracciones (Klein y Cruz-Uribe, 1984) teniendo en cuenta lateralidad, estado de fusión y tamaño relativo de los especímenes. Luego se calculó el Número Mínimo de Unidades Anatómicas (MAU), que posteriormente fue estandarizado en una escala de 0 a 100 (\%MAU) (Binford, 1978, 1984). El MNE fue utilizado para calcular las Partes Esqueletarias Básicas (PEB) (Yacobaccio, Madero, Malmierca y Reigadas, 1997-1998). Las PEB se calculan sumando las porciones de cada MNE agrupado en diez zonas del esqueleto: cabeza (cráneo y mandíbula), columna (vértebras cervicales, torácicas, lumbares y sacro), costillar (costillas y esternebras), pelvis, escápula, pata delantera (húmero y radioulna), pata trasera (fémur y tibia), pie delantero (carpianos y metacarpo), pie trasero (tarsianos y metatarso) y falanges. A partir de esta medida es posible observar la representación de las zonas esqueletarias, que se corresponden con la forma en que los pastores actuales trozan los animales (Yacobaccio et al., 1997-1998).

Hay varias maneras de medir la utilidad económica de las diferentes partes anatómicas que conforman una carcasa. Por un lado, resulta de interés destacar algunos índices que se basan en el consumo inmediato y jerarquizan los distintos huesos evaluando el potencial de carne y médula que ofrecen y su empleo al poco tiempo de que el animal murió. Para ello se utiliza la propuesta de Mengoni Goñalons (2013), que divide el esqueleto en 5 categorías (huesos con solo carne, huesos con mucha carne y médula, huesos con poca carne y médula, huesos con solo médula y huesos con seso). En cuanto al consumo diferido, se considera qué partes son las mejores para ser conservadas por desecación. Para ello se evalúa el índice de secado (alto, moderado alto, moderado bajo, bajo). Ambos indicadores se calcularon sobre el NISP. 


\section{Cerámica}

Con el objetivo de complementar la información brindada por el análisis de la muestra zooarqueológica y, principalmente, poder interpretar el origen de los depósitos que conformaron el registro del recinto 1 , se tendrá en cuenta una de las variables medidas en el análisis del material cerámico, es decir, el tamaño de los fragmentos (Berardi, 2004; Vaquer, 2011). La muestra está compuesta por 468 fragmentos cerámicos.

Es posible dividir a los factores que afectan la distribución espacial y la preservación de materiales en varios tipos, siendo los que nos ocupan aquí los factores físicos como la gravedad, la acción del agua y los tipos de superficies sedimentarias (contextos de erosión o depositación). Considerando cada fragmento cerámico como un tipo de clasto, es posible que el agrupamiento sea el resultado de la acción de diversos agentes. Mientras más grande sea el tamaño registrado se requerirá un agente con mayor fuerza para mover el fragmento. Para medir el tamaño de los fragmentos, utilizamos una escala derivada del análisis sedimentológico en la que cada tamaño es el doble en la escala (Berardi, 2004). El valor de esta clasificación es que permite generar interpretaciones sobre los posibles agentes que contribuyeron a la formación de los conjuntos. Se toma como hipótesis de trabajo que, a mayor tamaño del fragmento, mayor es la fuerza del agente para moverlo.

\section{Resultados}

\section{Óseo}

\section{Modificaciones óseas}

En primer lugar, se presentan algunas tendencias generales del análisis de la muestra y luego por conjunto arqueofaunístico. Como ya se ha mencionado, el NSP total de la muestra es de 1.590 especímenes óseos, de los que pudieron ser identificados (NISP) un total de 662 (42\%), contabilizando el resto de los fragmentos, es decir 928, como NID (58\%).

Los análisis de meteorización generales de la muestra muestran que, a partir del NISP\%, el $1 \%$ se encuentra en el estadio 1 de la escala de Behrensmeyer (1978), el 10\% en el estadio 2 , el $40 \%$ en el estadio 3, el $35 \%$ en el estadio 4 y el $14 \%$ en el 5 . Esta tendencia se mantiene en cada uno de los tres componentes que corresponden a los tres momentos de ocupación del recinto.

Para dar cuenta de la alteración térmica, se registró el color de los especímenes óseos del Número Total de Especímenes Óseos (NSP = NISP + NID). En los 3 conjuntos los resultados presentan diferencias. En el C1, el 71,79\% $(n=28)$ se encuentra calcinado. En el C2, más de la mitad de los especímenes se encuentran carbonizados $(\mathrm{n}=139)$, mientras que el $30 \%(\mathrm{n}=77)$ no presenta ningún signo de alteración térmica. En cambio, en el C3, el $71 \%(n=921)$ no presenta alteración térmica y el $24 \%$ se encuentra entre los estadios carbonizado y calcinado $(\mathrm{n}=316)$.

Se analizaron marcas de origen antrópico en todos los especímenes óseos relacionadas al procesamiento de los animales. Tanto en el C2 como en el C3 se registraron marcas en especímenes óseos de Artiodactyla y Camelidae. Teniendo en cuenta la total ausencia de cérvidos en el conjunto y que el subconjunto Artiodactyla está representado por huesos largos indeterminados y astillas de huesos largos, es posible plantear la hipótesis de que se trata de camélidos. En ambos conjuntos, los huesos que presentan algún tipo de marca no llegan al $4 \%$ de la muestra. Se registraron 14 especímenes con marcas de corte, uno con marca de machacado, uno con negativos de impacto y tres especímenes que presentan formatización. 
Entre los tres especímenes óseos que presentan formatización, se encuentra un hueso largo de artiodáctilo pulido de ambas caras formando una superficie cóncava. Las puntas se encuentran fracturadas. Los otros dos especímenes formatizados no pudieron ser identificados ni taxonómica ni anatómicamente. Ambos son huesos a los que se le dio una forma cónica y presentan marcas que lo circundan (Figura 5). Aún no se han analizado en relación a su funcionalidad. El resto de los taxones no presentó marcas de procesamiento. Tampoco se han registrado marcas de modificaciones óseas producidas por agentes no antrópicos.

\section{Diversidad taxonómica}

El conjunto presenta una baja diversidad taxonómica. El taxón más abundante fue Artiodactyla, representando el $45,47 \%(\mathrm{n}=301)$. El subconjunto Camelidae es el segundo taxón más representado con un $36,55 \%(\mathrm{n}=242)$. Además, se encuentran representados otros taxa en porcentajes menores (Tabla 1).

En los tres conjuntos se observa una predominancia de Artiodactyla y de Camelidae, constituyendo un $80 \%$ del NISP en cada conjunto. Sin embargo, en el C3 se observa, además de un incremento sustancial en la cantidad de especímenes óseos, cómo cobra mayor importancia la presencia de Chinchillidae (10,23\%).

\section{Identificación interespecífica de camélidos}

Con respecto a la identificación interespecífica de camélidos, solo se han hallado tres falanges proximales en el C3. Se registraron dos medidas de cada una de las falanges proximales: ancho máximo y espesor máximo de la superficie articular. Los valores métricos de los especímenes presentan una distribución polarizada entre los dos extremos del continuum. Dos de ellos se ubican cercanos a los valores correspondientes a llamas actuales, mientras que el restante se encuentra próximo (aunque por debajo, siendo un espécimen aún más pequeño) a los valores de vicuña actuales. La muestra total es demasiado pequeña para poder proponer hipótesis acerca del uso y consumo de animales silvestres.

\section{Representación de partes esqueletarias de camélidos}

Si tomamos en cuenta la composición general de la muestra y basándonos en el NISP, podemos observar que tanto el esqueleto axial como el esqueleto apendicular se encuentran representados por igual. El esqueleto axial presenta un mayor número de especímenes óseos, pero la diferencia no es significativa y puede deberse a la mayor posibilidad de fragmentación de esta zona del esqueleto. Ahora bien, si consideramos el MNE, la representación del esqueleto apendicular es significativamente mayor $(70,59 \%)$ con respecto al esqueleto axial (29,41\%). En el C1 los especímenes óseos identificados con Camelidae son demasiado escasos $(n=2)$ para ser tomados en cuenta. Por esta razón a continuación, se trabajará solo sobre el C2 y el C3.

En el C2 se observa la ausencia de varios elementos óseos correspondientes al esqueleto axial, como ser cráneo, mandíbula y dientes. Sin embargo, tanto cuando observamos el NISP como el MNE, no existe diferencia en la representación del esqueleto axial y el apendicular. En el C3, en cambio, se encuentran representadas todas las partes esqueletarias. Si se considera el NISP, se observa una leve predominancia del esqueleto axial $(57,21 \%)$ sobre el apendicular $(42,79 \%)$. En cambio, si se considera el MNE, la representación del esqueleto axial $(33,8 \%)$ es relativamente menor a la del esqueleto apendicular $(66,29 \%)$, lo que probablemente se relacione con una menor fragmentación 


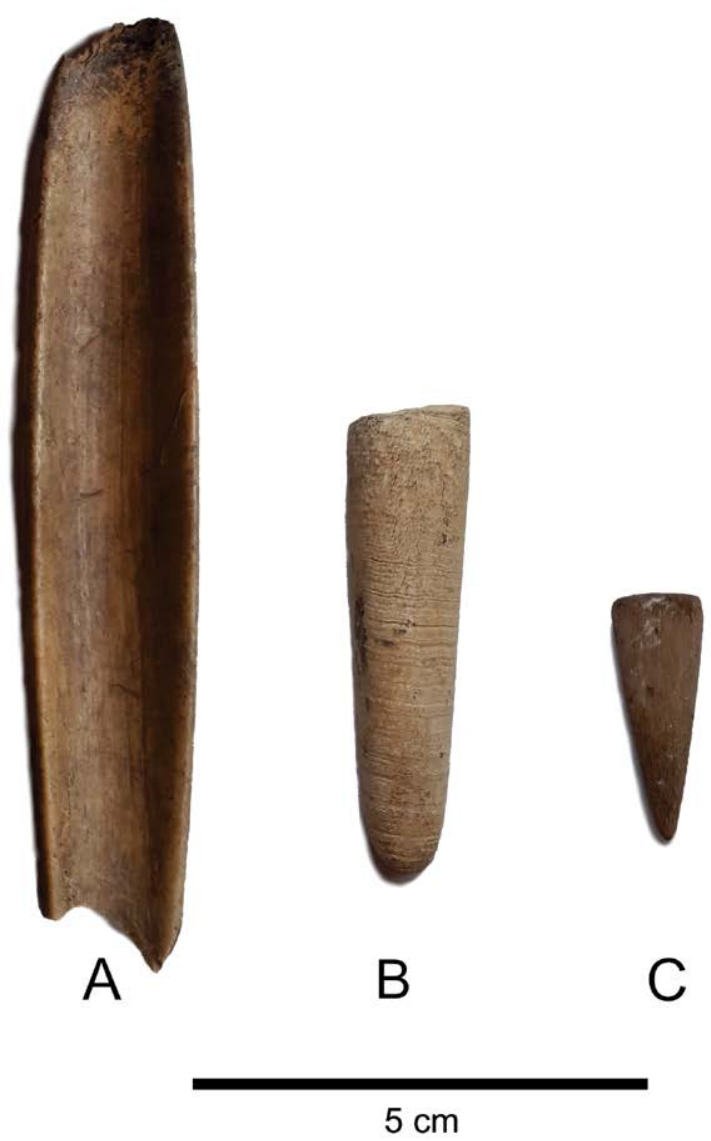

Figura 5. A) hueso largo de Artiodactyla formatizado. B y C) especímenes óseos no identificados formatizados.

\begin{tabular}{|l|c|c|c|c|c|c|}
\hline \multirow{2}{*}{ Taxón } & \multicolumn{2}{|c|}{ C1 $_{\mathbf{1}}$} & \multicolumn{3}{c|}{ C2 $_{2}$} & \multicolumn{2}{c|}{ C $_{3}$} \\
\cline { 2 - 7 } & NISP & \% & NISP & \% & NISP & $\%$ \\
\hline Artiodactyla & 9 & 81,82 & 32 & 43,24 & 260 & 45,05 \\
Camelidae & 2 & 18,18 & 33 & 44,59 & 207 & 35,88 \\
Chinchillidae & - & - & 5 & 6,76 & 59 & 10,23 \\
Rodentia & - & - & 4 & 5,41 & 50 & 8,67 \\
Avis & - & - & - & - & 1 & 0,17 \\
\hline Total NISP & $\mathbf{1 1}$ & $\mathbf{1 0 0 \%}$ & $\mathbf{7 4}$ & $\mathbf{1 0 0 \%}$ & $\mathbf{5 7}$ & $\mathbf{1 0 0 \%}$ \\
\hline
\end{tabular}

Tabla 1. Composición taxonómica de la muestra.

del esqueleto apendicular. Mientras que en el C2 fémur y tibia poseen el MAU más elevado, en el C3 corresponde al astrágalo (Tabla 2).

Si consideramos los huesos agrupados por Partes Esqueletarias Básicas (PEB) (Yacobaccio et al., 1998), en ambos conjuntos se observa una predominancia del esqueleto axial sobre el apendicular, pero la diferencia no es marcada. En el C2 registramos una mayor representación de la columna, mientras que en el C3 observamos una mayor presencia de falanges y en segundo lugar de la columna. 


\begin{tabular}{|c|c|c|c|c|c|c|c|c|}
\hline \multirow{2}{*}{ Parte esqueletaria } & \multicolumn{4}{|c|}{$\mathrm{C}_{2}$} & \multicolumn{4}{|c|}{$\mathrm{C}_{3}$} \\
\hline & NISP & MNE & MAU & MAU\% & NISP & MNE & MAU & MAU\% \\
\hline Cráneo & - & - & - & - & 28 & 2 & 2 & 80 \\
\hline Mandíbula & - & - & - & - & 8 & 2 & 1 & 40 \\
\hline Incisivo indeterminado & - & - & - & - & - & - & - & - \\
\hline Molar indeterminado & - & - & - & - & - & 1 & - & - \\
\hline Diente indeterminado & - & - & - & - & 16 & - & - & - \\
\hline Hiodes & - & - & - & - & - & - & - & - \\
\hline Atlas & - & - & - & - & - & - & - & - \\
\hline Axis & - & - & - & - & 1 & 1 & 1 & 40 \\
\hline Cervicales & 3 & 1 & 0,14 & 40 & 4 & 3 & 0,43 & 17,2 \\
\hline Torácicas & 2 & 1 & 0,08 & 16 & 5 & 3 & 0,25 & 10 \\
\hline Lumbares & 3 & 3 & 0,43 & 86 & 4 & 3 & 0,43 & 17,2 \\
\hline Vértebra indeterminado & 3 & 3 & - & - & 7 & 5 & - & - \\
\hline Costillas & 5 & 2 & 0,08 & 16 & 38 & 8 & 0,33 & 13,2 \\
\hline Pelvis & - & - & - & & 4 & 2 & 1 & 40 \\
\hline Escápula & - & - & - & - & 3 & 2 & 1 & 40 \\
\hline Húmero & - & - & - & - & 2 & 1 & 0,5 & 20 \\
\hline Radioulna & 3 & 1 & 0,5 & 100 & 2 & 2 & 1 & 40 \\
\hline Carpianos & 3 & 3 & 0,21 & 42 & 8 & 8 & 0,57 & 22,8 \\
\hline Fémur & 2 & 1 & 0,5 & 100 & 1 & 1 & 0,5 & 20 \\
\hline Tibia & 1 & 1 & 0,5 & 100 & 3 & 1 & 0,5 & 20 \\
\hline Tarsianos & 2 & 2 & 0,2 & 40 & 7 & 7 & 0,7 & 28 \\
\hline Astrágalo & - & - & - & - & 5 & 5 & 2,5 & 100 \\
\hline Calcáneo & - & - & - & - & 4 & 3 & 1,5 & 60 \\
\hline Metapodio & 2 & 1 & 0,25 & 50 & 16 & 4 & 1 & 40 \\
\hline Falange 1 & - & - & - & - & 16 & 10 & 1,25 & 50 \\
\hline Falange 2 & 2 & 1 & 0,13 & 26 & 11 & 7 & 0,88 & 35,2 \\
\hline Falange 3 & 2 & 2 & 0,25 & 50 & 4 & 4 & 0,5 & 20 \\
\hline Sesamoideos & - & - & - & - & 4 & 4 & - & - \\
\hline Total esqueleto axial & 16 & 10 & - & - & 115 & 30 & - & - \\
\hline $\begin{array}{l}\text { Total esqueleto } \\
\text { apendicular }\end{array}$ & 17 & 11 & - & - & 86 & 59 & - & - \\
\hline Total & 143 & 22 & - & - & 201 & 89 & - & - \\
\hline
\end{tabular}

Tabla 2. NISP, MNE, MAU y \%MAU de cada parte esqueletaria de Camelidae de los conjuntos arqueofaunísticos de los conjuntos 2 y 3.

Utilidad económica de partes esqueletarias de camélidos

Consideramos la utilidad económica de las diferentes partes anatómicas representadas en los conjuntos, teniendo en cuenta que los animales son potenciales proveedores de productos como carne, grasa, médula (productos que para obtenerlos es necesario matar al animal) y productos como fibra, transporte (para los que es necesario mantenerlo vivo) (Mengoni Goñalons, 2013). Tanto en el C2 como en el C3 predominan aquellos huesos que solo tienen carne (vértebras, costillas, pelvis y escápula) seguidos por aquellos 
que solo presentan médula (metapodios, falanges y calcáneo). En el C2 siguen de cerca los huesos que tienen poca carne y poca médula (radioulna y tibia) y en C3 aquellos que tienen seso (cabeza). Por último, en ambos conjuntos se encuentran pobremente representados aquellos huesos con mucha carne y médula (húmero y fémur) (Tabla 3).

Esta información se complementa con aquella que obtuvimos al examinar las frecuencias de las diferentes partes con el marco del índice de secado. Los dos conjuntos presentan diferencias. Por un lado, en el C2 predominan las partes que poseen un índice de secado moderado/alto (vértebras y pelvis, $42,31 \%$ ), seguidos por aquellos que poseen un índice de secado bajo (huesos largos, falanges y cráneo, 38,46\%). Por otro lado, en el C3 se observa una predominancia de aquellas partes que presentan un bajo índice de secado (51,63\%), seguidos de aquellos que poseen un alto índice (costillas y esternebras, $24,84 \%)$. Entonces, en el C2 alrededor del $60 \%$ de los restos corresponden a huesos que poseen un alto índice de secado, seguidos por aquellos que poseen un índice de secado bajo (40\%). En el C3, el 60\% de los restos corresponden a huesos con un índice de secado bajo y el $40 \%$ a un índice de secado alto entre alto y moderado (Tabla 4 ).

\section{Perfiles etarios}

En relación con la construcción de perfiles etarios, se tuvo en cuenta la fusión ósea. Así, la construcción de los perfiles se realizó a partir de las frecuencias de NISP -F o NF- para cada una de las tres etapas anteriormente mencionadas. Debido a que el C1 presenta muy pocos especímenes, aquí presentaré los resultados del C2 y el C3.

En el C2, se puede observar que en la fusión temprana todos los especímenes registrados sobrevivieron a los 18 meses de edad. En la intermedia se reparten los porcentajes entre animales que murieron antes de los 18-36 meses y los que sobrevivieron a esa edad. Por último, en la etapa de fusión tardía, el 100\% supera los 48 meses de vida.

En el C3 se ve que, en la etapa de fusión temprana, el 100\% sobrevivió a los 18 meses de edad, igual que sucede en la etapa intermedia. Por último, en fusión tardía, observamos que casi la totalidad de los especímenes registrados sobrevive a los 48 meses de edad. Respecto al análisis de las piezas dentarias, todas se registraron sueltas y mal conservadas, por lo que no fueron consideradas para el cálculo etario de Camelidae.

\section{Cerámica}

Como ya se ha explicitado, aquí solo se presentarán los resultados de los análisis realizados sobre fragmentos cerámicos con el objetivo de complementar la información de la historia posdepositacional del recinto 1 de PJN. Sobre un total de 448 fragmentos, la mayoría se encuentra dentro del Tamaño 2 (212: 47\%) seguido por el Tamaño 3 (144: 32\%). No registramos material de Tamaño 5, y el Tamaño 4 se encuentra representado por solamente 41 fragmentos ( $9 \%$ del conjunto). Esto permite interpretar que la mayoría de los fragmentos se encuentra dentro de las clases de tamaño que pueden ser movilizadas por agentes como el agua. Si se considera que un recinto es un ambiente de depositación puntual (Butzer, 1982), donde los muros funcionan como trampas de material, se puede determinar que el conjunto procede del recinto mismo. A su vez, el recinto se encuentra en una posición topográfica elevada en relación con su entorno inmediato, por lo que no sería un ambiente de depositación de material proveniente de afuera. Sin embargo, la ausencia de materiales de tamaños mayores indica que los fragmentos no se encuentran in situ, con excepción de un cántaro subglobular compuesto alisado recuperado enterrado en el piso del recinto. Es posible determinar que, si bien los fragmentos no proceden de otro lugar, su distribución dentro del recinto es el producto de agentes posdepositacionales, por lo que no es posible reconocer áreas de actividad específicas. 


\begin{tabular}{|l|c|c|c|c|c|}
\hline \multirow{2}{*}{ Utilidad } & \multirow{2}{|c|}{ C $_{2}$} & \multicolumn{2}{|c|}{ C $_{3}$} \\
\cline { 2 - 5 } & Partes anatómicas & NISP & NISP\% & NISP & NISP\% \\
\hline Huesos con solo carne & vértebras, costillas, pelvis y escápula & 16 & $61,55 \%$ & 66 & $43,14 \%$ \\
Huesos con mucha carne y médula & húmero, fémur & 2 & $7,69 \%$ & 3 & $1,96 \%$ \\
Huesos con poca carne y médula & radioulna, tibia, mandíbula & 4 & $15,38 \%$ & 13 & $8,50 \%$ \\
Huesos con solo médula & metapodios, calcáneo, falanges 1 y falange 2 & 4 & $15,38 \%$ & 43 & $28,10 \%$ \\
Huesos con seso & cráneo & 0 & $0 \%$ & 28 & $18,30 \%$ \\
\hline NISP Total & - & 26 & $\mathbf{1 0 0 \%}$ & $\mathbf{1 5 3}$ & $\mathbf{1 0 0 \%}$ \\
\hline
\end{tabular}

Tabla 3. Partes esqueletarias de camélidos e índice de utilidad económica de partes esqueletarias de los conjuntos 2 y 3.

\begin{tabular}{|l|c|c|c|c|c|}
\hline \multirow{2}{*}{ Índice de secado } & \multirow{2}{*}{ Partes anatómicas } & \multicolumn{2}{|c|}{ C2 $_{2}$} & \multicolumn{2}{c|}{ C $_{3}$} \\
\cline { 3 - 6 } & costillas y esternebras & NISP & NISP\% & NISP & NISP\% \\
\hline Alto & vértebras y pelvis & 5 & $19,23 \%$ & 38 & $24,84 \%$ \\
Moderado-alto & mandíbula y escápula & 11 & $42,31 \%$ & 25 & $16,34 \%$ \\
Moderado-bajo & huesos largos, falanges (1 y 2) y cráneo & 10 & $0,00 \%$ & 11 & $7,19 \%$ \\
Bajo & - & $\mathbf{2 6}$ & $\mathbf{1 0 0 \%}$ & $\mathbf{1 5 3}$ & $\mathbf{1 0 0 , 0 0 \%}$ \\
\hline NISP Total & & & & & \\
\hline
\end{tabular}

Tabla 4. Partes esqueletarias de camélidos e índice de secado de los conjuntos 2 y 3.

\section{Discusión y comentarios finales}

Como se ha planteado al inicio de este trabajo, es importante remarcar que estos incipientes resultados solo permiten interpretar las primeras tendencias en relación con la obtención, manejo, aprovechamiento y consumo de animales en PJN durante las tres ocupaciones identificadas. Se considera que, en primer lugar, y en consonancia con uno de los objetivos de esta investigación, es importante tener en cuenta la formación del registro arqueológico y poder identificar aquellos procesos posdepositacionales que tuvieron lugar en el recinto 1 de este sitio. A partir de cruzar la información obtenida del análisis cerámico con los estadios de meteorización presentados previamente, se puede interpretar que los depósitos de la cuadrícula 1 son el producto de las actividades realizadas en el recinto. Sin embargo, los materiales recuperados no se encuentran in $s i t u$, sino que fueron trabajados por diferentes agentes antes de su enterramiento. Esto se evidencia en los estadios altos de meteorización del conjunto zooarqueológico y el tamaño de los fragmentos del material cerámico. La baja presencia de Rodentia y la ausencia de otros indicadores como excremento indican una baja incidencia de los roedores como agentes posdepositacionales.

Es importante volver a señalar que el recinto 1 de PJN se encuentra emplazado en un sitio compuesto por pocos recintos habitacionales, estructuras agrícolas y de manejo del agua y posibles corrales. Esto nos da la pauta de que las prácticas alimenticias están relacionadas tanto con la cría, aprovechamiento y consumo de animales como con la producción agrícola. Varios autores remarcan como una característica intrínseca del sistema económico pastoril, la falta de autarquía y la necesidad de integración con otras formas de producción (Galaty y Johnson, 1990; Nielsen, 2000; Yacobaccio et al., 1997-1998). Entonces, resulta consecuente que los resultados obtenidos a partir del 
análisis de la muestra zooarqueológica nos revelen una tendencia en relación con el aprovechamiento de recursos en algunos aspectos similar a la encontrada en pastores actuales (Capriles, 2014, 2017; Kuznar, 1995; Tomka, 1994; Yacobaccio, 1994; Yacobaccio et al., 1998; Yacobaccio y Madero, 2001) aunque las formas de complementariedad varíen. Es importante tener en cuenta que el sistema de asentamiento pastoril actual es complejo y está compuesto por diferentes unidades residenciales que no permanecen ocupadas a lo largo de todo el año (Göbel, 2002). En el estado actual de las investigaciones en la región resulta difícil tener en cuenta este factor como influyente en la conformación del registro arqueológico.

A partir de los resultados obtenidos, se presentan a continuación las tendencias generales identificadas en el análisis del registro zooarqueológico. En los dos bloques temporales que tuve en cuenta (ya que la ocupación superficial presenta solo 39 especímenes óseos), los camélidos constituyen los animales con mayor representación en toda la muestra. Hay que remarcar que aquellos especímenes asignados a artiodáctilos también fueron contabilizados como camélidos debido a la ausencia de cérvidos en la muestra. Ahora bien, la muestra analizada es muy pequeña para permitir interpretar las estrategias vinculadas al aprovechamiento de animales silvestres en función la identificación interespecífica de camélidos. Sin embargo, ya que se ha identificado un espécimen que correspondería a un camélido de tamaño pequeño (posiblemente correspondiente a Vicugna vicugna), se puede hipotetizar acerca del consumo de este tipo de animales. Considero que teniendo en cuenta el contexto y la importancia de este tipo de recursos en varios sitios registrados en Puna, el consumo de animales silvestres pudo haber complementado el consumo de animales domésticos (Lama glama principalmente) como parte de los recursos que formaron parte de las prácticas alimentarias (Escola, 2002).

Por otro lado, si se analizan los perfiles etarios se puede interpretar aquellas estrategias adoptadas con respecto a los camélidos domésticos. El predominio general de especímenes fusionados, alcanzando casi el $90 \%$ en los espacios domésticos para las categorías de fusión intermedia y tardía, se encuentra en consonancia con la información etnoarqueológica proveniente de estrategias de manejo de recursos de pastores actuales. Una de las interpretaciones de esta información está relacionada con el sacrificio de animales adultos. Esta estrategia responde a las características de sistemas productivos mixtos, como por ejemplo aquellos orientados a la producción no especializada de fibra y carne (Capriles, 2014; Göbel, 2001; Maryañski, 2013; Nielsen, 2000, 2001; Yacobaccio, 2007).

En la muestra analizada, en las tres categorías de fusión ósea predominan los huesos fusionados, la mayoría entre los estadios de fusión intermedia y tardía. Esto puede deberse a que posiblemente estaban siendo criados para acceder a los productos que como ser la fibra y la capacidad de carga, para los que se precisa mantener vivos a los animales. Por otro lado, advertimos un patrón de aprovechamiento integral de camélidos completos. Se observa que se han descartado las diferentes unidades anatómicas de acuerdo con las necesidades de consumo de los habitantes del recinto. Aunque los porcentajes de representación de partes esqueletarias básicas varíen, las diferencias en las representaciones no son marcadas. Podría suponerse que ambos conjuntos zooarqueológicos son el resultado de actividades de sacrificio, procesamiento, consumo y descarte in situ. Sin embargo, para tratarse de un recinto de habitación doméstico, resulta llamativa la cantidad de restos óseos hallados. En general suele ser esperable encontrar un número bajo de especímenes en estos ámbitos, ya que la limpieza periódica del espacio y la posibilidad de utilizar áreas externas donde descartar los residuos, disminuyen notablemente el número de restos óseos. En este caso, el NISP cuenta 1.590 huesos, constituyendo un número relativamente importante (Maryañski, 2013; Yacobaccio et al., 1998). 
Teniendo en cuenta que han sido identificadas dos ocupaciones permanentes, me interesa comparar las prácticas de consumo. En base a los datos presentados, se puede observar que no existen diferencias marcadas que permitan suponer cambios en las prácticas relacionadas con la esfera de la comida entre una ocupación y la otra. Más bien, parece existir una continuidad en la manera en la que se manejaron y consumieron los camélidos. Es interesante que los dos fogones registrados se encuentran en la primera ocupación del recinto (correspondiente al C3) y que en aquella ocupación más tardía no hemos registrado aún estructuras de combustión. Esto puede deberse a que la muestra aún está sesgada, ya que se han analizado los materiales provenientes de la mitad del recinto. Además, más del $70 \%$ de los especímenes correspondientes a C2 presentan algún tipo de evidencia de alteración térmica. Otra alternativa es que existieran efectivamente cambios en algunas de las prácticas relacionadas con la producción de alimentos, y las estructuras de combustión se encontraran en alguno de los espacios exteriores, extra muros del recinto.

Como he mencionado en la introducción, la forma en la que los alimentos han sido cocinados en el pasado es un fenómeno complejo que está determinado por diversos factores. En líneas generales, la obtención de los ingredientes ha sido objeto de estudio arqueológico, mientras que la cocina se ha dejado de lado. La práctica de cocinar es central en la vida diaria de las personas que habitaron estos espacios. Considero que el análisis de los restos faunísticos, junto con estudios morfológico-funcionales de la cerámica, el análisis de microvestigios vegetales y el estudio de las estructuras de combustión, son todas líneas de evidencia que pueden otorgarnos información acerca de este "acto sensorial total" (Le Breton, 2007, p. 263) de la comida y de la cocina. Sin embargo, lamentablemente aún no tenemos desarrollado el análisis del resto de los materiales desde el punto de vista de su relación y función dentro de las prácticas culinarias, por lo que nos queda un largo camino por recorrer con este objetivo en mente. Este trabajo constituye un primer acercamiento hacia una "arqueología de la comida" en la zona de estudio (Pazzarelli, 2008). Entendemos, sin embargo, que la comprensión de las prácticas culinarias no se agota con el estudio del registro zooarqueológico.

Por último, resulta interesante considerar la presencia de tres huesos formatizados dentro del conjunto analizado. Si bien aún no he profundizado en el análisis funcional de estos, su presencia nos permite hipotetizar sobre prácticas de producción ósea que escapan de la esfera de las prácticas alimentarias y del contexto de producción y preparación de comida, otorgándonos la idea de que los huesos fueron considerados también como materia prima y no solo como residuos. Ya hemos continuado con la excavación de la mitad restante del recinto, por lo que, a futuro, planificamos analizar esta muestra y de esta manera ampliar las interpretaciones del sitio. Junto con esto, considero que es necesario cruzar otras líneas de evidencia que nos permitan ahondar en la comprensión de las prácticas de preparación de comida en contextos domésticos de la región, ya que la zooarqueología nos permite acceder a una porción incompleta del fenómeno multidimensional de la alimentación.

\section{Agradecimientos}

Esta investigación se encuentra financiada por el sistema de becas doctorales del Consejo Nacional de Investigaciones Científicas y Técnicas. Agradezco a la Comunidad Aborigen "Orqo Runas" y a la Comisión Municipal de Cusi Cusi por el apoyo brindado en las tareas de campo. A Laura Pey por la confección de los mapas y planos del sitio. Finalmente, agradezco a los evaluadores anónimos por sus aportes para mejorar el manuscrito de este trabajo. 


\section{Referencias citadas}

》 Albeck, M. y Ruiz, M. (2003). El Tardío en la Puna de Jujuy. Poblados, etnías y territorios. Cuadernos de la Facultad de Humanidades y Ciencias Sociales de la Universidad Nacional de Jujuy, 20, 199-219.

"Ávido, D. (2012). Conceptos y métodos para el estudio zooarqueológico de la cocción de los alimentos. En M. P. Babot, M. Marschoff y F. Pazzarelli (Eds.), Las manos en la masa. Arqueologías, Antropologías e Historias de la Alimentación en Suramérica (pp. 553-574). Córdoba: Universidad Nacional de Córdoba.

»Behrensmeyer, A. (1978). Taphonomic and Ecologic Information from Bone Weathering. Paleobiology, 4, 150-162.

" Berardi, M. (2004). Historia Ocupacional de Los Amarillos (Quebrada de Yacoraite, Jujuy). Análisis del material arqueológico en superficie mediante SIG. (Tesis de Licenciatura inédita), Universidad de Buenos Aires, Argentina.

" Binford, L. (1978). Nunamiut Ethnoarchaeology. Nueva York: Academic Press.

》Binford, L. (1984). Faunal Remains from Klasies River Mouth. Orlando: Academic Press.

» Buitrago, L. (1999). El clima de la provincia de Jujuy. San Salvador de Jujuy: Facultad de Ciencias Agrarias, Universidad Nacional de Jujuy.

"Butzer, K. (1982). Archaeology as Human Ecology. Cambridge: Cambridge University Press.

"Cabrera, A. L. (1976). Regiones fitogeográficas argentinas. En W.F. Kluger (Ed.), Enciclopedia Argentina de Agricultura y Jardinería (Tomo II, pp. 1-85). Buenos Aires: Acme.

"Capriles, J. M. (2014). Mobile communities and pastoralist landscapes during the Formative period in the Central Altiplano of Bolivia. Latin American Antiquity, 25 (1), 3-26.

"Capriles, J. M. (2017) Arqueología del pastoralismo temprano de camélidos en el Altiplano Central de Bolivia. La Paz: Institut Français d'Études Andines (IFEA).

" De Feo, C., Fernández, A. y Raviña, M. (2001). Abra De Lagunas. Un asentamiento Tardío en la porción Noroccidental de la Puna Jujeña. En Actas del XIV Congreso Nacional de Arqueología (pp. 1-6). Rosario: Universidad Nacional de Rosario.

» De Feo, C., Fernández, A. y Raviña, M. (2007). Las cabeceras del Río Grande de San Juan y sus relaciones con áreas vecinas durante los últimos momentos del Desarrollo Cultural Prehispánico. Cuadernos de la Facultad de Humanidades y Ciencias Sociales de la Universidad Nacional de Jujuy, 32, 135-149.

»Elkin, D. (1995). Volume density of South American camelid skeletal parts. International Journal of Osteoarchaeology, 5, 29-37.

»Escola, P. (2002). Caza y pastoralismo: un reaseguro para la subsistencia. Relaciones de la Sociedad Argentina de Antropología, XXVII, 233-245.

» Galaty, J. G. y Johnson, D. L. (1990). The world of pastoralism. Nueva York y Londres: Guilford/ Belhaven.

" Göbel, B. (2001). El ciclo anual de la producción pastoril en Huancar (Jujuy, Argentina). En G. Mengoni, D. Olivera y H. Yacobaccio (Eds.), El uso de los camélidos a través del tiempo (pp. 91-115). Buenos Aires: Ediciones del Tridente.

"Göbel, B. (2002). La arquitectura del pastoreo: uso del espacio y sistema de asentamientos en la Puna de Atacama (Susques). Estudios Atacameños, 23, 53-76. 
"Goody, J. (1995). Cocina, cuisine y clase. Estudio de sociología comparada. Barcelona: Gedisa.

»Izeta, A. (2007). Zooarqueología del sur de los Valles Calchaquíes (provincias de Catamarca y Tucumán, República Argentina): Análisis de conjuntos faunísticos del primer milenio A.D. Oxford: BAR International Series, Archaeopress.

» Izeta, A., Cattáneo, R., Scattolin, M. y Cortés, L. (2013). Changed into tools. Camelid bones from the Southern Calchaquíes Valleys (Formative Period, North-western Argentina). En A. Choyke y S. O'Connor (Eds.), From these Bare Bones: Raw Materials and the Study of Worked Osseous Objects (pp. 50-58). Oxford: Oxbow Books.

» Klein, R. y Cruz-Uribe, K. (1984). The analysis of animal bones from archaeological sites. Chicago: University of Chicago Press.

» Kuznar, L. (1995). Awatimarka. The ethnoarchaeology of an Andean herding community. California: Wadsworth Inc Fulfillment.

»Le Breton, D. (2007). El sabor del mundo. Una antropología de los sentidos. Buenos Aires: Nueva Visión.

» Lyman, R. L. (1994). Vertebrate taphonomy. Cambridge: Cambridge University Press.

» Marschoff, M. (2007). ¿Comer o nutrirse? La alimentación como práctica social. Arqueología, 13, 155-184.

» Maryañski, J. (2013). Arqueofaunas de un espacio doméstico e implicancias para el pastoreo prehispánico en el Río Grande de San Juan. La Zaranda de Ideas, 9(2), 103-117.

" Mengoni Goñalons, G. L. (1999). Cazadores de guanacos en la estepa patagónica. Buenos Aires: Sociedad Argentina de Antropología.

» Mengoni Goñalons, G. L. (2013). El aprovechamiento de la fauna en sociedades complejas: aspectos metodológicos y su aplicación en diferentes contextos arqueológicos del NOA. En V. Williams y M. B. Cremonte (Comps.), Al Borde del Imperio. Paisajes Sociales, Materialidad y Memoria en Áreas Periféricas del Noroeste Argentino (pp. 311-396). Buenos Aires: Sociedad Argentina de Antropología.

» Miyano, J. P. (2018). El uso de animales por las sociedades agropastoriles tempranas: análisis zooarqueológico de un basural de la aldea de Palo Blanco (Valle de Fiambalá, Catamarca). Arqueología, 24, 77-101.

"Nielsen, A. (2000). Andean caravans: An ethnoarchaeology. (Tesis Doctoral inédita), University of Arizona, Estados Unidos.

"Nielsen, A. (2001). Ethnoarchaeological perspectives on caravan trade in the SouthCentral Andes. En L. Kuznar (Ed.), Ethnoarchaeology of Andean South America: Contributions to archaeological method and theory (pp. 163-201). Oxford y Nueva York: Berghahn Series.

» Nielsen, A., Ávalos, J., Ávila, F., Guagliardo, J. P. y López, M. (2008). Reapertura de las investigaciones arqueológicas en San Juan Mayo. En Actas de las IX Jornadas Regionales de Investigación en Humanidades y Ciencias Sociales (p. 219). San Salvador de Jujuy: Facultad de Humanidades y Ciencias Sociales, Universidad Nacional de Jujuy.

" Pazzarelli, F. (2008). Notas acerca de una arqueología de la comida. La Zaranda de Ideas, 4, 157-162.

»Pey, L. (2015). Lógica agrícola y espacialidad en la Quebrada de Pajchela (Rinconada, Puna de Jujuy): el caso de Casas Quemadas. En Libro de resúmenes de las $X$ Jornadas de Jóvenes Investigadores en Ciencias Antropológicas (pp. 78-79). Buenos Aires: Instituto Nacional de Antropología y Pensamiento Latinoamericano. 
»Samuel, D. (1996). Approaches to the archaeology of food. Petits Propos Culinaires, 54, 12-21.

»Saravia, T. (1960). Geografía de la provincia de Jujuy. Buenos Aires: Instituto Geográfico Militar.

» Tomka, S. (1994). Quinua and camelids on the Bolivian Altiplano: An ethnoarchaeological approach to agro-pastoral subsistence production with an emphasis on agro-pastoral transhumance. (Tesis Doctoral inédita), University of Texas, Estados Unidos.

»Vaquer, J. M. (2011). Paisaje, materialidad y prácticas sociales en Cruz Vinto. Saarbrücken: Editorial Académica Española.

»Vaquer, J. M., Gerola, I. Carboni, B. y Bonelli, J. (2014). Cazadores, pastores y agricultores. Lógicas del paisaje en Cusi-Cusi, Cuenca Superior del Río San Juan Mayo (Jujuy, Argentina). En M. Beierlein y D. Gutiérrez (Eds.), Desarrollos Regionales (1000-1500 DC) en el Sur de Bolivia y el Noroeste Argentino (pp. 30-46). Tarija: La Pluma del Escribano.

» Vaquer, J. M., Zuccarelli, V., Pey, L. y Cámera, Y. (2014). Paisajes agrícolas de la dominación y sus relaciones interregionales: el caso de Casas Quemadas (Cuenca Superior del Río San Juan Mayo, Jujuy, Argentina. En M. Beierlein y D. Gutiérrez (Eds.), Desarrollos Regionales (1000-150o DC) en el Sur de Bolivia y el Noroeste Argentino (pp. 47-63). Tarija: La Pluma del Escribano.

"Von den Driesch, A. (1976). A guide to the measurement of animal bones from archaeological sites. Bulletin I. Cambridge: Peabody Museum of Archaeology and Ethnology.

» Yacobaccio, H. (1994). Etnoarqueología de pastores surandinos: Una herramienta para conocer el registro arqueológico. En H. Yacobaccio y C. Madero (Eds.), Jornadas de Arqueología e Interdisciplinas (pp. 203-236). Buenos Aires: Programa de Estudios Prehistóricos, CONICET.

» Yacobaccio, H. (2007). Andean camelid herding in the South Andes: Ethnoarchaeological models for archaeozoological research. Anthropozoologica, 42(2), 143-154.

» Yacobaccio, H. y Madero, C. (2001). Ethnoarchaeology of a pastoral settlement of the Andean Plateau: An investigation of archaeological scale. En L. Kuznar (Ed.), Ethnoarchaeology of Andean South America: Contributions to archaeological method and theory (pp. 84-96). Oxford y Nueva York: Berghahn.

» Yacobaccio, H., Madero, C. y Malmierca, M. (1998). Etnoarqueología de pastores surandinos. Buenos Aires: Grupo de Zooarqueología de Camélidos.

» Yacobaccio, H., Madero, C., Malmierca, M. y Reigadas, M. (1997-1998). Caza, domesticación y pastoreo de camélidos en la Puna Argentina. Relaciones de la Sociedad Argentina de Antropología, XXII-XXIII, 389-418. 